\title{
Dynamical and Steady State Processes of Probe Absorption-Dispersion in a Four-Level Quantum Dot Nanostructure
}

\author{
E. Asadi Amirabadi ${ }^{a}$, M. JAmshidnejAd ${ }^{b}$, S. Miraboutalebi ${ }^{b}$ And S.H. Asadpour ${ }^{c, *}$ \\ ${ }^{a}$ Young Researchers and Elite Club, Marvdasht Branch, Islamic Azad University, Marvdasht, Iran \\ ${ }^{b}$ Department of Physics, Faculty of Science, Islamic Azad University, North Tehran Branch, Tehran, Iran \\ ${ }^{c}$ Department of Physics, Marvdasht Branch, Islamic Azad University, Marvdasht, Iran
}

(Received April 11, 2016; in final form January 12, 2017)

\begin{abstract}
In this paper, we investigated the transient electron population and the transient behaviour of the dispersion, absorption and refractive property of weak probe light in a four-level InGaN/GaN quantum dot nanostructure. In order to achieve the wave functions and their corresponding energy levels of the mentioned quantum dot nanostructure, the Schrödinger and Poisson equations is solved selfconsistently for carriers (here electron) in quantum dot. Our findings show that the properties of transient processes can be dramatically affected by parameters such as intensity, detuning and relative phase of applied fields. Our proposed scheme provides a realistic model for transient control of refraction index properties in a quantum dot nanostructure. These results may have potential applications in high speed optical switch for quantum information technologies.
\end{abstract}

DOI: 10.12693/APhysPolA.131.1460

PACS/topics: quantum dot nanostructure, absorption, dispersion, optical transient processes

\section{Introduction}

It is well known that optical properties of coherent medium can be considerably qualified by application of external fields. Quantum coherence and quantum interference are the basic mechanisms for qualifying the reaction of the atomic medium to the applied fields [1]. Many theoretical computations of different nonlinear optical phenomena in different atomic system have been studied by many research groups in two past decades [1-12]. One of the most important aspects of these properties is the modification of the absorption, dispersion, and nonlinearity of the system. Due to interaction of optical fields with nonlinear media, many interesting phenomena such as electromagnetically induced transparency $[2,3]$, lasing without inversion (LWI) [4], high refractive index without absorption [5, 6], four-wave mixing [7], optical solitons [8], optical bistability $[9,10]$ and so on $[11,12]$ have been investigated. Moreover, experimental evolution of above nonlinear optical phenomena has been reported and discussed. For example, light propagating in a $\mathrm{Rb}$ atoms has been analyzed experimentally by $\mathrm{Ba}-$ jcsy et al. [13]. Experimental observations of interference between three-photon and one-photon excitations, and phase control of light attenuation in a four-level atomic system have been reported [14]. Similar phenomena based on the quantum interference and coherence in semiconductor quantum wells (SQWs) and quantum dots (QDs) have also been extensively studied in recent

\footnotetext{
* corresponding author; e-mail:

s.hosein.asadpour@gmail.com, S.Hosein.Asadpour@miau.ac.ir
}

years [15-23]. The theory of quantum coherence phenomena in SQDs was introduced by Chow et al. on the basis of earlier studies [24-26].

It is well known that the devices based on intersubband transitions in the SQWs and QDs have many inherent advantages rather than atomic systems $[27,28]$. Therefore, similar phenomena based on the quantum interference and coherence have also been extensively studied in recent years, such as lasing without inversion [29], EIT [30], optical bistability and multistability [17,31], the Kerr nonlinearity [20], optical solitons [32, 33], four-wave mixing [34] and others [19, 22, 35] in SQWs and QDs.

The effect of quantum coherence on transient behaviors of solid state medium are widely investigated recently in SQWs and QDs [36-45]. Furthermore, recent experimental studies have used transient absorption for the study of optical effects in intersubband transitions in SQWs [46, 47].

To the best of our knowledge, the transient properties of four-level closed atomic media are rarely investigated in InGaN/GaN QD. In this paper, due to scientific principle in quantum optics and solid material, we investigate the transient electron population and the transient features of the dispersion, absorption and refractive property of InGaN/GaN quantum dot nanostructure.

The impact of the Rabi frequency of the driving field and relative phase between applied fields on temporal features of the dispersion and absorption are then discussed. Based our recent work [23], we propose a four-level In$\mathrm{GaN} / \mathrm{GaN}$ quantum dot nanostructure model by solving the Schrödinger and Poisson equations self-consistently.

Our proposed scheme is based on Ref. [48], but our equations and results are drastically different from that work. First, we are interested in studying the dynamical 
properties of refractive index in a four-level InGaN/GaN quantum dot nanostructure. Second, we find that the relative phase of applied fields has essential roles to controlling of transient refractive property of quantum dot nanostructure. Third, the dynamical behaviour of enhancing in refractive index of quantum dot is accompanied by lasing without inversion in the medium. The dynamical response of the system is important due to its novel application in high speed optical switch that is consequential technique for quantum computing, quantum information network, and communication.

\section{Model and equations}

A four-level InGaN/GaN quantum dot nanostructure based Ref. [23] has been considered for studying the dynamical properties of refractive index and optical transient processes. The quantum dot dimension information for calculating the wave functions and eigenenergies are listed in Table I which results from Ref. [23]. It is emphasized that these properties result from solving the Schrödinger-Poisson equations selfconsistently for carrier in InGaN/GaN quantum dot's conduction band. The normalized wave functions with related eigenenergies are displayed in Fig. 1. By consideration of these properties one can obtain the properties of laser fields for interacting by four-level quantum dot nanostructure. The configuration of InGaN/GaN quantum dot nanostructure is presented in inset of Fig. 1b.

TABLE I

Quantum dot dimensions for calculating the wave functions and eigenenergies.

\begin{tabular}{c|c}
\hline \hline InGaN/GaN & Material \\
\hline $37 \AA$ & radius of quantum dot \\
$25 \AA$ & quantum dot barrier \\
$0.6 \mathrm{~V}$ & applied external voltage \\
$70 \AA$ & length of quantum dot \\
4 & obtaining energy levels
\end{tabular}

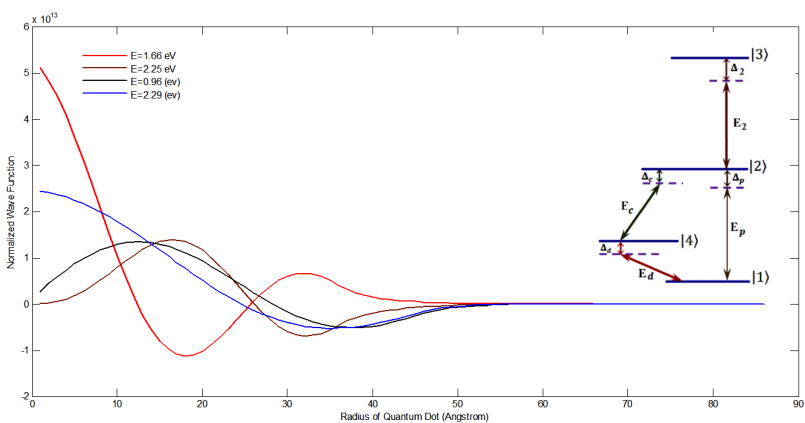

Fig. 1. (a) Wave functions of 4-level nanocrystal. Radius of quantum dot $37 \AA$, the thickness of shell (barrier) $25 \AA$. (b) Schematic diagram of 4-level InGaN/GaN quantum dot nanostructure interacted with a weak probe light, a coherent coupling and pumping fields and a cycling field.
The transition $|2\rangle \leftrightarrow|4\rangle$ can be mediated by using a strong coupling field $E_{c}$ and frequency $\omega_{c}$. A pumping field $E_{2}$ (frequency $\omega_{2}$, phase $\phi_{2}$ ) and a weak probe light (frequency $\omega_{p}$, phase $\phi_{p}$ ) are coupled transitions $|3\rangle \leftrightarrow|2\rangle$ and $|2\rangle \leftrightarrow|1\rangle$, respectively. The field $E_{d}$ (frequency $\omega_{d}$, phase $\phi_{d}$ ) is applied to the transition $|4\rangle \leftrightarrow|1\rangle$. The corresponding Rabi frequencies for the respective transitions are defined as: $\Omega_{c}=\mu_{24} E_{c} / 2 \hbar$, $\Omega_{2}=\mu_{32} E_{2} / 2 \hbar, \Omega_{p}=\mu_{21} E_{p} / 2 \hbar$, and $\Omega_{d}=\mu_{41} E_{d} / 2 \hbar$. The master equation of motion for the density operator in an arbitrary multilevel atomic system can be written as

$$
\frac{\partial \rho}{\partial t}=-\frac{\mathrm{i}}{\hbar}[H, \rho]+L_{\rho} .
$$

The term $L_{\rho}$ represents the incoherent pumping and decay part in the system, where $L$ is the Liouvillian operator acting on the density matrix $\rho$. Using the definitions of the density matrix and the expression for the Hamiltonian for the four-level atom, and after carrying out the rotating wave approximation, we get the following set of equations for the time evolution of the system:

$$
\begin{aligned}
& \dot{\rho}_{11}(t)=-\mathrm{i}\left(\Omega_{p} \rho_{12}+\Omega_{d} \rho_{14}\right)+2 \gamma_{4} \rho_{44}+2 \gamma_{1} \rho_{22}+\text { H.c. } \\
& \dot{\rho}_{33}(t)=\mathrm{i} \Omega_{2} \rho_{23}-2 \gamma_{2} \rho_{33}+\text { H.c., } \\
& \dot{\rho}_{44}(t)=-\mathrm{i}\left(\Omega_{c} \rho_{42}-\Omega_{d} \rho_{14}\right)+2 \gamma_{3} \rho_{22}-2 \gamma_{4} \rho_{44}+\mathrm{H} . c . \\
& \dot{\rho}_{12}(t)=-\mathrm{i} \Omega_{p}^{*}\left(\rho_{11}-\rho_{22}\right)-\mathrm{i} \Omega_{2} \rho_{13}-\mathrm{i} \Omega_{c}^{*} \rho_{14} \\
& \quad-\left(\gamma_{1}+\gamma_{3}-\mathrm{i} \Delta_{p}\right) \rho_{12}+\mathrm{i} \Omega_{d}^{*} \rho_{42}, \\
& \dot{\rho}_{13}(t)=\mathrm{i} \Omega_{d}^{*} \rho_{43}-\mathrm{i} \Omega_{2}^{*} \rho_{12}+\mathrm{i} \Omega_{p}^{*} \rho_{23} \\
& \quad-\left(\gamma_{2}-\mathrm{i} \Delta_{p}-\mathrm{i} \Delta_{2}\right) \rho_{13}, \\
& \dot{\rho}_{14}(t)=-\mathrm{i} \Omega_{d}^{*}\left(\rho_{11}-\rho_{44}\right)+\mathrm{i} \Omega_{p}^{*} \rho_{24}-\mathrm{i} \Omega_{c} \rho_{12} \\
& \quad-\left(\gamma_{4}-\mathrm{i} \Delta_{p}+\mathrm{i} \Delta_{c}\right) \rho_{14}, \\
& \dot{\rho}_{23}(t)=\mathrm{i} \Omega_{2}^{*}\left(\rho_{33}-\rho_{22}\right)+\mathrm{i} \Omega_{p} \rho_{13}+\mathrm{i} \Omega_{c} \rho_{43} \\
& \quad-\left(\gamma_{3}+\gamma_{1}+\gamma_{2}-\mathrm{i} \Delta_{2}\right) \rho_{23}, \\
& \dot{\rho}_{24}(t)=\mathrm{i} \Omega_{c}\left(\rho_{44}-\rho_{22}\right)+\mathrm{i} \Omega_{p} \rho_{14}-\mathrm{i} \Omega_{d}^{*} \rho_{21}+\mathrm{i} \Omega_{2}^{*} \rho_{34} \\
& \quad-\left(\gamma_{1}+\gamma_{3}+\gamma_{4}+\mathrm{i} \Delta_{c}\right) \rho_{24}, \\
& \dot{\rho}_{34}(t)=\mathrm{i} \Omega_{2} \rho_{24}-\mathrm{i} \Omega_{c} \rho_{32}-\mathrm{i} \Omega_{d}^{*} \rho_{31} \\
& \quad-\left(\gamma_{2}+\gamma_{4}+\mathrm{i} \Delta_{c}+\mathrm{i} \Delta_{2}\right) \rho_{34} .
\end{aligned}
$$

The above density matrix elements additionally obey the normalization and Hermitian condition $\sum_{i=1}^{3} \rho_{i i}=1$ and $\rho_{i j}=\rho_{j i}^{*}$. With the initial conditions $\rho_{11}(0)=1$ and other $\rho_{i j}(0)=0(i j=1,2,3)$ the following discussions will be deployed based on the time-dependent numerical solutions of Eq. (2), where $\Delta_{p}=\omega_{p}-\omega_{21}, \Delta_{c}=\omega_{c}-\omega_{24}$, $\Delta_{2}=\omega_{2}-\omega_{32}$ and $\Delta_{d}=\omega_{d}-\omega_{41}$ are the detunings of the corresponding incident lights. Since the three driving fields form a closed-loop configuration, the phase can be inserted into any one of them, and it will not change the results of the calculation. In other words, if all the fields had been phase dependence, only the collection phase would be important and no individual 
phase-dependent term would occur. This collective phase can be easily determined to be $\Phi=\phi_{2}+\phi_{d}-\phi_{c}$ by repeating the coherence term calculation $\rho_{21}$ and noting that the Rabi frequencies are complex in general. Here $\phi_{i}$ is the phase of the complex Rabi frequency $\Omega_{i}$ of the driving fields, and $\omega_{21}, \omega_{32}, \omega_{24}$ and $\omega_{41}$ are the resonant frequencies which associated with the corresponding optical transitions. The total population and dephasing decay rates $2 \gamma_{i}(i=1,2,3,4)$ are added phenomenologically in the density matrix equations [28, 35, 49], which are comprised of a population decay contribution as well as a dephasing contribution. The first contribution is mainly due to longitudinal-optical (LO) phonon emission events at low temperature and the dephasing contribution may originate from electron-electron scattering and electron-phonon scattering as well as inhomogeneous broadening due to scattering on interface roughness [28, 35, 49]. The set of Eqs. (2) can be solved numerically to obtain the transient response of the medium. It is well known that the absorption and dispersion are related to the susceptibility of the system. Therefore, the probe absorption-dispersion coefficient coupled to the transitions $|2\rangle \leftrightarrow|1\rangle$ is proportional to the term $\rho_{21}$. The imaginary parts $\operatorname{Im} \rho_{21}$ correspond to gainabsorption coefficient, while the real parts $\operatorname{Re} \rho_{21}$ relates to dispersion coefficient. If $\operatorname{Im} \rho_{21}>0$, the probe field will be absorbed; on the contrary, the probe field will be amplified. In the following section, we analyze the numerical results of Eqs. (2) for discussing the transient features of the absorption and the dispersion.

\section{Results and discussion}

It is known that the linear response of the system as well as absorption, refraction, and group velocity is governed by complex polarization $\rho_{21}$. In fact, the medium responsibility to the applied fields is shown by the susceptibility $\chi$, which can be defined as:

$$
\chi=\frac{2 N}{\varepsilon_{0} E_{p}} \mu_{21} \rho_{21} .
$$

For further discussion the relevant quantity to consider is the group index, $n_{g}=\frac{c}{v_{g}}$, where $c$ is the speed of light in vacuum and the group velocity $v_{g}$ is given by

$$
v_{g}=\frac{c}{1+2 \pi \operatorname{Re} \chi\left(\omega_{p}\right)+2 \pi \omega_{p} \frac{\partial \chi\left(\omega_{p}\right)}{\partial \omega_{p}}} .
$$

Equations (2), (3) and (4) are our basic results for the discussion of absorption, dispersion and group velocity of probe light. It is known that the group velocity of a probe light depends strongly on the slope of the dispersion. It is shown that the slope of dispersion will be changed from positive to negative by intensity of the cycling field and relative phase of applied fields. Moreover, we find that the enhanced refractive index in a region accompanied with probe amplification. Enhancing in the refractive index with amplification is favorable for us when the pulse passes through the medium. In the following, a few numerical results under some parametric conditions are presented when the refractive index enhanced. In our calculations the imaginary part of $\rho_{21}$ corresponds to the single-photon absorption and $\rho_{33}$ corresponds to the twophoton absorption for the probe field, respectively. For the steady-state behavior, we plot $\operatorname{Im} \rho_{21}$ or $\rho_{33}$ versus probe detuning $\Delta_{p}$, while for the transient behavior we plot $\operatorname{Im} \rho_{21}$ versus time.

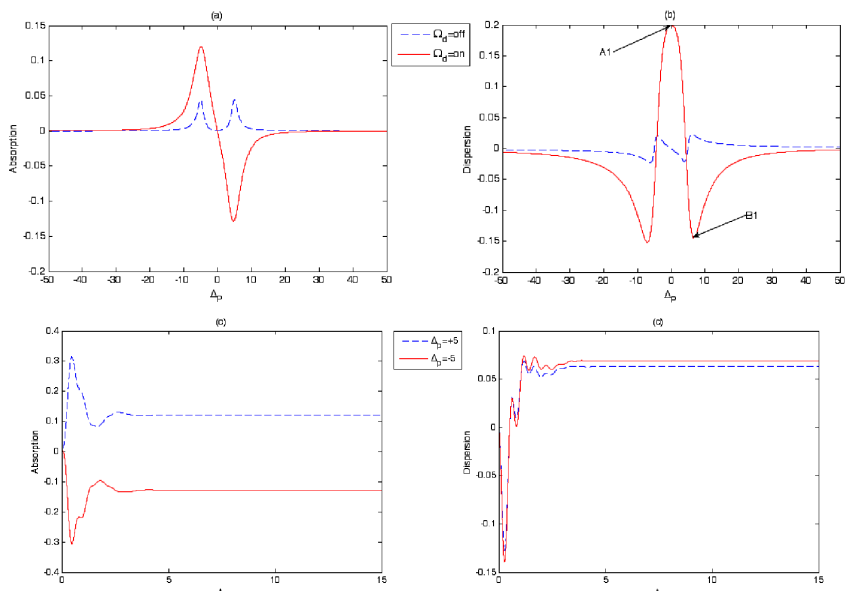

Fig. 2. Absorption (a), dispersion (b) of weak probe light versus detuning in the absence (dashed line) and presence (solid line) of cycling field. Time evolution of the probe absorption (c), dispersion (d) in the presence of cycling field: solid line, $\Delta_{p}=-5$; and dashed line, $\Delta_{p}=+5$. The selected parameters are $\Omega_{c}=5 \mathrm{meV}$, $\Omega_{2}=0.2 \mathrm{meV}, \Omega_{p}=0.1 \mathrm{meV}, \Delta_{i}=0, \Phi=0, \gamma_{1}=$ $\gamma_{2}=\gamma_{3}=1 \mathrm{meV}, \gamma_{4}=0.2 \mathrm{meV}$.

These numerical results are shown in Figs. 2-9. The steady-state and transient behaviors of weak probe light absorption and dispersion in the absence and presence of cycling field are presented in Fig. 2. It is realized that, in the absence of cycling field $\left(\Omega_{d}=0\right)$, the index of refraction with zero absorption cannot be obtained. In this case, the group index of weak probe field is equal to zero. However, due to presence of cycling field (solid line), the index of refraction with zero absorption is obtained. Therefore, the refractive index can be enhanced via adjusting the intensity of cycling field. Here, large refractive index with zero and negative absorption are located at points $A_{1}$ and $B_{1}$. The temporal features of absorption and dispersion of weak probe light are displayed in Fig. 2c,d. It can be seen that for $\Delta_{p}=-5$ (solid line), the transient absorption decreases rapidly and finally reaches a negative value. By changing the detuning $\Delta_{p}=+5$ (dashed line), the absorption starts to increase as the time increases, and finally reaches to negative and positive absorption at the steady state, respectively. In the following, we show the effects of the relative phase between applied fields on steady-state and transient behaviors of inverted Y-type four-level InGaN/GaN quantum dot nanostructure. In the presence of cycling field, the closed-loop configuration makes this medium become phase dependent. 


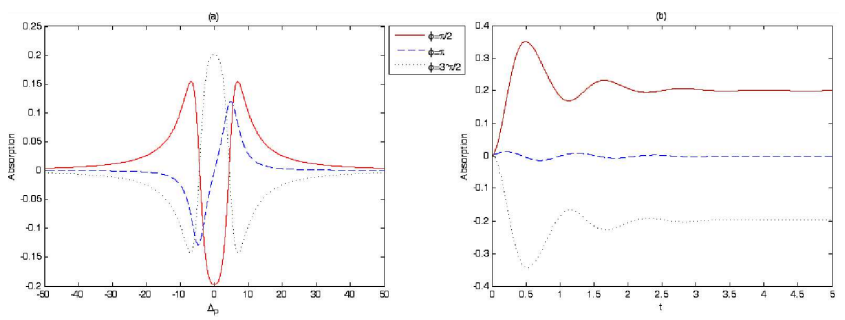

Fig. 3. Absorption of weak probe light versus detuning (a) for relative phase $\Phi=\pi / 2$ (solid line), $\Phi=\pi$ (dashed line) and $\Phi=3 \pi / 2$ (dotted line). Time evolution of the probe absorption (b) for relative phase $\Phi=\pi / 2$ (solid line), $\Phi=\pi$ (dashed line) and $\Phi=3 \pi / 2$ (dotted line). The selected parameter are $\Delta_{p}=0$ and other are the same as in Fig. 2.
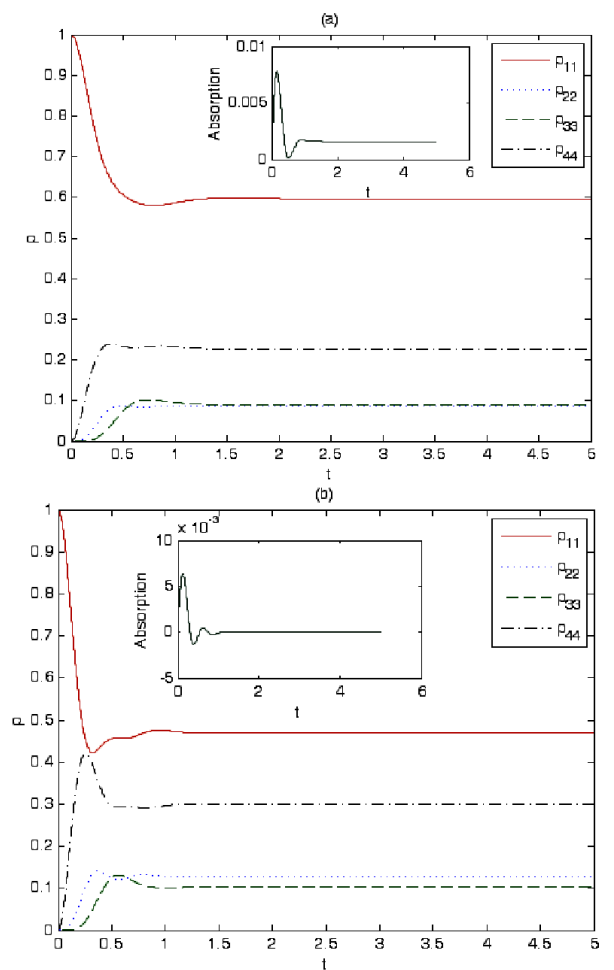

(c)

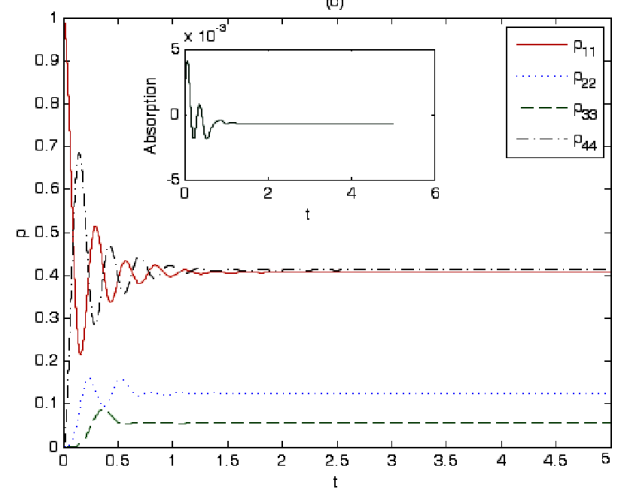

Fig. 4. Time evolution of the population in the fourlevel atomic system with absorption probe light versus time for $\Omega_{d}=3 \mathrm{meV}$ (a), $\Omega_{d}=5 \mathrm{meV}$ (b) and $\Omega_{d}=$ $10 \mathrm{meV}$ (c). The selected parameters are $\Omega_{2}=5 \mathrm{meV}$, $\gamma_{1}=4 \mathrm{meV}, \gamma_{2}=3 \mathrm{meV}, \gamma_{4}=2 \mathrm{meV}$ and other are the same as in Fig. 2.

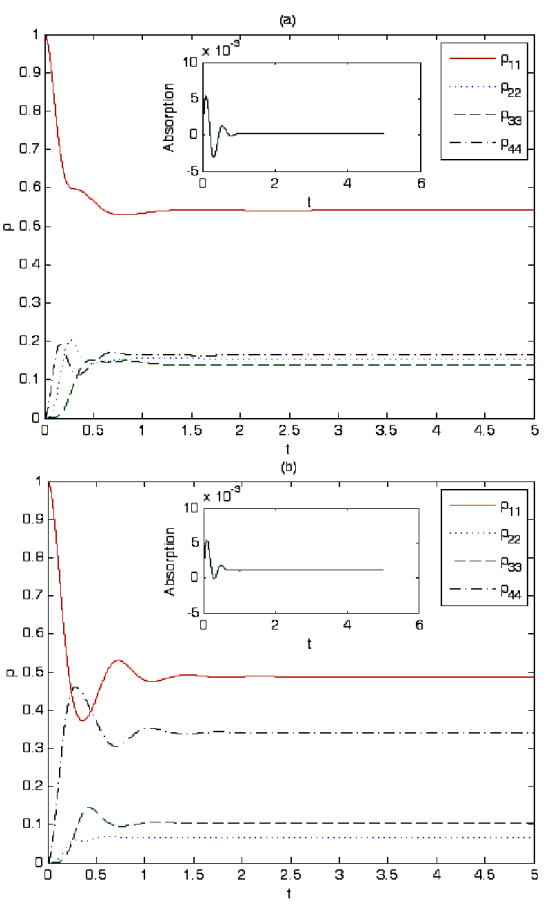

Fig. 5. Time evolution of the population in the fourlevel atomic system with absorption probe light versus time for $\Omega_{d}=\Omega_{2}=5 \mathrm{meV}, \Omega_{c}=10 \mathrm{meV}$ (a), $\Omega_{d}=$ $\Omega_{c}=5 \mathrm{meV}, \Omega_{2}=10 \mathrm{meV}$ (b). The other selected parameters are the same as in Fig. 4.

Therefore, the relative phase $\Phi$ can affect the absorption properties of probe light which is shown in Fig. 3. In Fig. 3a we present the steady-state absorption spectrum versus $\Delta_{p}$ for different values of phase $\Phi$. It is clearly seen that the absorption spectrum is sensitive to the relative phase between applied fields. Transient behavior of the absorption for different values of phase $\Phi$ is shown in Fig. 3b. It can be seen that by increasing the relative phase the absorption changes from positive to the negative (dashed and dotted lines). In this case, probe absorption is vanished and converts to the probe gain (dotted line). This means that the transient behaviors of the probe field can be tuned by changing the relative phase of applied fields.

In Figs. 4 and 5, we show the time evolution of the population distribution in states $|1\rangle,|2\rangle,|3\rangle$ and $|4\rangle$ for the different values of the Rabi frequencies of the applied fields. It can be found that in first condition the probe absorption increases for a short time, then steeply descends and eventually reaches a positive constant. In this case, most of the population remains in level $|1\rangle$. Therefore, the probe field experiences absorption, and population inversion does not appear.

The transient behaviors of absorption-dispersion for different values of cycling field is discussed in Fig. 6a and $\mathrm{b}$. It is found that at $t=0, \Omega_{d}=5 \mathrm{meV}$ (solid line) and $\Omega_{d}=10 \mathrm{meV}$ (dashed line) the probe absorption is zero, once the time increases the absorption oscillates and finally reaches to the steady-state values. We can conclude that by increasing the intensity of the cycling field, 
the absorption decreases. As for $\Omega_{d}=5 \mathrm{meV}$ (solid line), the absorption oscillates and reaches to its positive value in steady-state condition. In this case the dispersion is negative, which corresponds to the superluminal light propagation. The dispersion properties of the probe field are very useful for its potential applications of controlling the group velocity of light from subluminal to superluminal. Our numerical calculation for the transient response shows that our system exhibits normal dispersion (or subluminal light) and exhibits anomalous dispersion (or superluminal light) by supplying the intensity of cycling field.

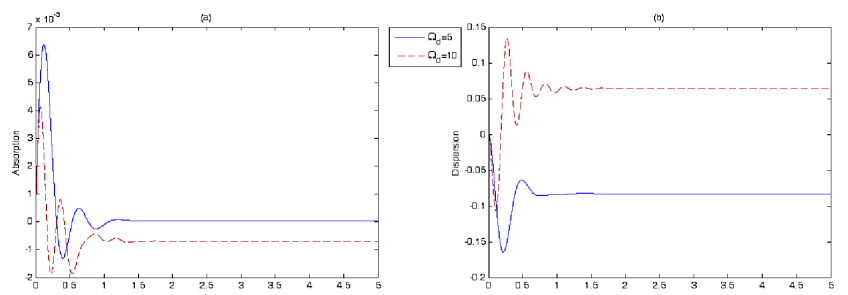

Fig. 6. Time evolution of the probe absorption (a), dispersion (b) for different values of the cycling field. Other parameters are the same as in Fig. 4.

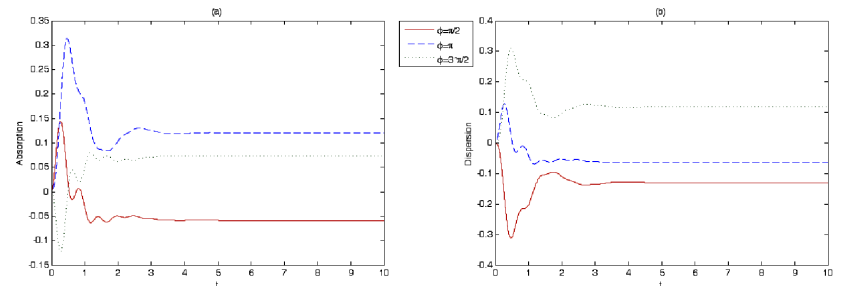

Fig. 7. Time evolution of the probe absorption (a), dispersion (b) for relative phase $\Phi=\pi / 2$ (solid line), $\Phi=\pi$ (dashed line) and $\Phi=3 \pi / 2$ (dotted line). The selected parameter are $\Delta_{p}=-5$ and other are the same as in Fig. 2.

The influence of relative phase on transient absorption and dispersion is displayed in Fig. 7. It is observed that the probe absorption-dispersion is completely phase dependent. At $t=0$, the absorption is zero, while by enhancing the time, absorption oscillates and finally reaches the steady-state value. The amount of probe absorption depends on the relative phase between the applied fields. The dispersion properties of the weak probe field is also phase dependent as shown in Fig. 7b. In addition, for $\Phi=\pi$ (dashed line) the steady state value of absorption coefficient is positive relating to probe absorption, while for $\Phi=\pi / 2$ (solid line) its value changes to negative that corresponds to a gain. In the next step, we present the dependence of the probe absorption-dispersion on the intensity of the cycling field under two-photon resonant condition.

In Fig. 8a, we find that the absorption coefficient on both sides of the spectrum increases when we increase the amplitude of the cycling field. Similar results are obtained for probe dispersion which is presented in Fig. 8b. At the end, we plot the absorption and dispersion properties of weak probe light versus relative phase of applied fields. It can be easily realized that the steady-state and transient behaviors of absorptive and refractive properties of weak probe light are dramatically sensitive to the cycling field and relative phase of applied fields.

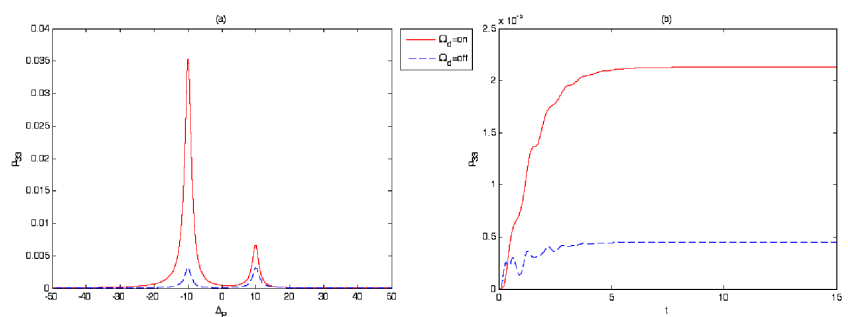

Fig. 8. Steady-state behavior of two-photon absorption $\rho_{33}$ versus probe detuning (a). Transient behavior of two-photon absorption $\rho_{33}$ (b). Dashed line, absence of cycling field; and solid line, presence of cycling field. Other parameters are the same as in Fig. 2.
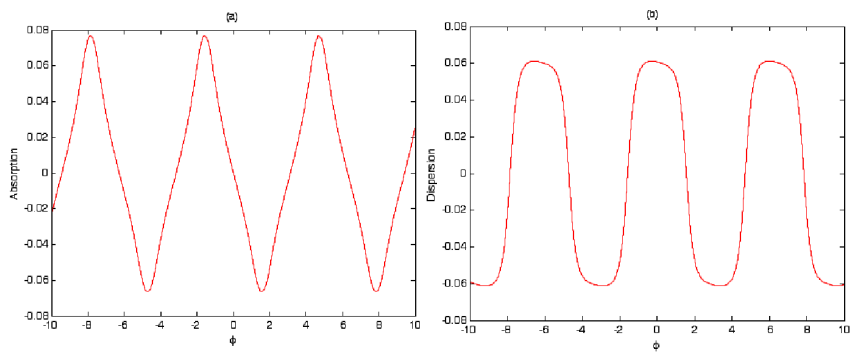

Fig. 9. Absorption (a) and dispersion (b) of weak probe light versus relative phase of applied fields. The selected parameter are $\Delta_{d}=5$. The other parameters are the same as in Fig. 2.

\section{Conclusion}

In summary, we have analyzed the effects of applied field on transient response of the absorptive, refractive and group index of weak probe field in an InGaN/GaN quantum dot nanostructure which were designed numerically by solving the Schrödinger and Poisson equations. We show that the intensity of cycling field and relative phase of applied fields can impact the transient and steady-state absorptive and refractive properties of a weak probe light dramatically. Also, we find that the intensity of cycling field and relative phase can be used to manipulation of enhanced refractive index with amplification at different detuning of probe light and transient and steady-state absorption and dispersion can be modulated by relative phase between applied fields. We hope that our proposed model may be useful for application in solid-state all-optical communication system by using a quantum dot nanostructure.

\section{References}

[1] Z. Ficek, S. Swain, Quantum Coherence and Interference: Theory and Experiments, Springer, Berlin 2004.

[2] S.E. Harris, Phys. Today 50, 36 (1997). 
[3] K.J. Boller, A. Imamoglu, S.E. Harris, Phys. Rev. Lett. 66, 2593 (1991).

[4] Y. Zhao, D. Huang, C. Wu, J. Opt. Soc. Am. B Opt. Phys. 13, 1614 (1996).

[5] M. Fleischhauer, C.H. Keitel, M.O. Scully, Phys. Rev. A 46, 1468 (1992).

[6] H.R. Hamedi, Gediminas Juzeliūnas, A. Raheli, M. Sahrai, Opt. Commun. 311, 261 (2013).

[7] Y. Wu, X. Yang, Phys. Rev. B 76, 054425 (2007).

[8] L.G. Si, W.X. Yang, X.Y. Lou, X.Y. Hao, Phys. Rev. A 82, 013836 (2010).

[9] J.H. Li, X.Y. Lou, J.M. Luo, Q.J. Huang, Phys. Rev. A 74, 035801 (2006).

[10] S.H. Asadpour, A. Eslami-Majd, J. Lumin. 132, 1477 (2012)

[11] Z. Wang, B. Yu, Laser Phys. Lett. 11, 035201 (2014).

[12] K.I. Osman, A. Joshi, Opt. Commun. 293, 86 (2013).

[13] M. Bajcsy, A.S. Zibrov, M.D. Lukin, Nature 426, 638 (2003).

[14] H. Kang, G. Hernandez, J. Zhang, Y. Zhu, Phys. Rev. A 73, 011802 (2006).

[15] S.M. Sadeghi, S.R. Leffler, J. Meyer, Opt. Commun. 151, 173 (1998).

[16] S.M. Sadeghi, S.R. Leffler, J. Meyer, Phys. Rev. B Condens. Matter 59, 15388 (1999).

[17] J.H. Li, Phys. Rev. B 75, 155329 (2007).

[18] Z. Wang, B. Yu, J. Opt. Soc. Am. B 30, 2915 (2013).

[19] S.H. Asadpour, M. Jaberi, H. Rahimpour Soleimani, J. Opt. Soc. B 30, 1815 (2013).

[20] S.H. Asadpour, H.R. Hamedi, A. Eslami-Majd, M. Sahrai, Physica E 44, 464 (2011).

[21] Y. Wu, J. Appl. Phys. 103, 104903 (2008).

[22] S.H. Asadpour, H. Rahimpour Soleimani, Opt. Commun. 315, 347 (2014).

[23] S.H. Asadpour, Z. Golsanamlou, H. Rahimpour Soleimani, Physica E 54, 45 (2013).

[24] J. Kim, O. Benson, H. Kan, Y. Yamamoto, Nature 397, 500 (1999).

[25] P. Michler, A. Kiraz, C. Becher, W.V. Schoenfeld, P.M. Petroff, L. Zhang, E. Hu, A. Imamoglu, Science 290, 2282 (2000).

[26] Y. Yamamoto, Phys. Rev. Lett. 89, 233602 (2002).

[27] S.H. Asadpour, H. Rahimpour Soleimani, Chin. Phys. Lett. 31, 114207 (2014).
[28] W.X. Yang, A.X. Chen, R.K. Lee, Y. Wu, Phys. Rev. A 84, 013835 (2011).

[29] C.R. Lee, Y.C. Li, F.K. Men, C.H. Pao, Y.C. Tsai, J.F. Wang, Appl. Phys. Lett. 86, 201112 (2005).

[30] A. Joshi, Phys. Rev. B 79, 115315 (2009).

[31] J.H. Li, Opt. Commun. 274, 366 (2007).

[32] W.X. Yang, J.M. Hou, R.K. Lee, Phys. Rev. A 77, 033838 (2008).

[33] W.X. Yang, R.K. Lee, Europhys. Lett. 83, 14002 (2008).

[34] X. Hao, J.H. Li, X. Yang, Opt. Commun. 282, 3339 (2009).

[35] Z. Wang, B. Yu, S. Zhen, X. Wu, J. Lumin. 134, 272 (2013).

[36] H. Jafarzadeh, A. Eslami-Majd, S.H. Asadpour, H. Rahimpour Soleimani, Optik 125, 1558 (2014).

[37] Z.H. Xiao, K. Kim, Opt. Commun. 282, 2547 (2009).

[38] M. Sahrai, A. Maleki, R. Hemmati, M. Mahmoudi, Eur. Phys. J. D 56, 105 (2010).

[39] Z.Q. Zeng, B.P. Hou, Optik 122, 1231 (2011).

[40] Z.Q. Zeng, B.P. Hou, Z.H. Gao, Y.P. Yang, Opt. Rev. 19, 45 (2012).

[41] Z. Zeng, Y. Wang, B. Hou, Z. Gao, Optik 124, 973 (2013).

[42] Zhi Qiang Zeng, Yu Ping Wang, Bang Pin Hou, Eur. Phys. J. D 67, 76 (2013).

[43] Zhiping Wang, Benli Yu, Xuqiang Wu, Shenglai Zhen, Zhigang Cao, Jun Zhu, Superlatt. Microstruct. 50, 734 (2011).

[44] S.G. Kosionis, A.F. Terzis, E. Paspalakis, J. Appl. Phys. 112, 073117 (2012).

[45] S.G. Kosionis, A.F. Terzis, E. Paspalakis, J. Lumin. 140, 130 (2013).

[46] M. Wagner, M. Helm, M.S. Sherwin, D. Stehr, Appl. Phys. Lett. 99, 131109 (2011).

[47] D. Dietze, J. Darmo, K. Unterrainer, Opt. Expr. 20, 23053 (2012)

[48] Z. Wang, H. Fan, J. Lumin. 130, 2084 (2010).

[49] Z. Wang, S. Zhen, X. Wu, J. Zhu, Z. Cao, B. Yu, J. Lumin. 143, 605 (2013). 